DRAFT VERSION NOVEMBER 3, 2018

Preprint typeset using LTEX style emulateapj v. 11/10/09

\title{
COMPARISON OF THE DIFFUSE H $\alpha$ AND FUV CONTINUUM BACKGROUNDS: ON THE ORIGINS OF THE DIFFUSE H $\alpha$ BACKGROUND
}

\author{
KWANG-IL SEON ${ }^{1}$, AdOLF WITT ${ }^{2}$, IL-JOONG KIM ${ }^{1}$, JONG-Ho SHINN ${ }^{1}$, \\ JERRY EDELSTEIN ${ }^{3}$, KYOUNG-WOOK MIN ${ }^{4}$, AND WONYONG HAN ${ }^{1}$ \\ Draft version November 3, 2018
}

\begin{abstract}

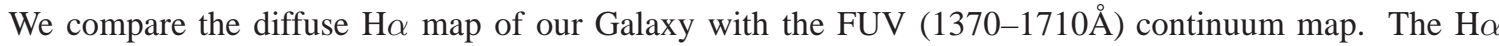
intensity correlates well with the FUV intensity. The $\mathrm{H} \alpha / \mathrm{FUV}$ intensity ratio increases in general with the H $\alpha$ intensity and the FUV hardness ratio (1370-1520 to $1560-1710 \AA$ ), implying that late OB stars may be the main source of the $\mathrm{H} \alpha$ recombination line at high latitudes. The variation of the $\mathrm{H} \alpha$ intensity as a function of the Galactic latitude is also very similar to that of the FUV intensity. The results likely suggest that not only the original radiation sources of the $\mathrm{H} \alpha$ and FUV backgrounds but also the radiative transfer mechanisms responsible for the diffuse backgrounds are largely common. Therefore, we propose a scenario wherein the $\mathrm{H} \alpha$ background at high latitudes is mostly composed of two components, $\mathrm{H} \alpha$ photons produced by in-situ recombination at the ionized regions around late $\mathrm{OB}$ stars and dust-scattered light of the $\mathrm{H} \alpha$ photons originating from late $\mathrm{OB}$ stars.
\end{abstract}

Subject headings: diffuse radiation — ISM: structure — ultraviolet: ISM

\section{INTRODUCTION}

The diffuse $\mathrm{H} \alpha$ background is generally believed to originate from the extended, nearly fully ionized regions (called warm ionized medium, or WIM) along the lines of sight (Reynolds et al. 1998; see Haffner et al. 2009 for a recent review). The volume filling fraction of the WIM increases from $\sim 0.1$ at the midplane to $>0.3-0.4$ at $|z|=1 \mathrm{kpc}$ (Kulkarni \& Heiles 1987; Reynolds 1991; Peterson \& Webber 2002). The large energy requirement of the WIM strongly suggests that $\mathrm{O}$ stars in the Galactic plane are the primary ionization source (Reynolds 1984, 1990a). There have been numerous investigations about how the Lyman continuum (Lyc) photons can escape the immediate vicinity of the $\mathrm{O}$ stars (or the bright $\mathrm{H}$ II regions) in the Galactic plane and ionize the diffuse interstellar medium (ISM) (Miller \& Cox 1993; Dove \& Shull 1994; Seon 2009a; Wood et al. 2010). However, the question of how the Lyc photons can propagate almost freely up to the scaleheight $(\sim 1$ $\mathrm{kpc}$ ) of the WIM has not been fully resolved because the photoionization models require the presence of very low density paths through the ISM.

In this paper, we explore two alternatives to explain the diffuse $\mathrm{H} \alpha$ emission of our Galaxy. The first scenario, which was investigated for external galaxies, is that late, field $\mathrm{OB}$ stars outside the bright $\mathrm{H}$ II regions ionize some of the WIM, reducing the required Lyc leakage from the bright $\mathrm{H}$ II regions in the galactic plane (Hoopes \& Walterbos 2000; Hoopes et al. 2001). The mean spectral type of stars in the field is later than that in H II regions (Massey et al. 1995; Hoopes \& Walterbos 2000). As the $\mathrm{H} \alpha$ intensity is directly proportional to Lyc intensity in the ionization bounded regions and the later-type

\footnotetext{
${ }^{1}$ Korea Astronomy and Space Science Institute, Daejeon 305-348, Republic of Korea; kiseon@kasi.re.kr

${ }^{2}$ Ritter Astrophysical Research Center, University of Toledo, Toledo, $\mathrm{OH}$, USA

${ }^{3}$ Space Sciences Laboratory, University of California, Berkeley, CA 94702, USA

${ }^{4}$ Korea Advanced Institute of Science and Technology, Daejeon 305701, Republic of Korea
}

stars would produce a lower ratio of ionizing Lyc to nonionizing far-ultraviolet (FUV) luminosities, the H $\alpha /$ FUV intensity ratio can constrain the spectral type of the ionizing stars. Hoopes \& Walterbos (2000) and Hoopes et al. (2001) measured $\mathrm{H} \alpha / \mathrm{FUV}$ intensity ratios for 10 nearby spiral galaxies, and concluded that late OB stars in the field are indeed an important source of ionization in those galaxies.

Secondly, we note that the majority of the $\mathrm{H} \alpha$ excess intensity in a number of high-latitude clouds in our Galaxy can be understood as light scattered off the interstellar dust (Mattila et al. 2007; Lehtinen et al. 2010; Witt et al. 2010). The possible presence of scattered $\mathrm{H} \alpha$ radiation at highlatitude clouds was first considered by Jura (1979). Photoionization by an external field of Lyc is expected to result in ionization of a thin outer shell of an isolated interstellar cloud while the observations of the $\mathrm{H} \alpha$ light from the high-latitude clouds clearly showed the dense inner core of the cloud as the brightest feature. Witt et al. (2010) argued that both a substantial fraction of the diffuse high-latitude $\mathrm{H} \alpha$ background intensity and much of the variance in the highlatitude $\mathrm{H} \alpha$ background are due to scattering by interstellar dust of $\mathrm{H} \alpha$ photons originating elsewhere in the Galaxy. If this is the case, the diffuse $\mathrm{H} \alpha$ emission should be closely related to the far-ultraviolet (FUV) continuum background, which is mostly dust-scattered light of the FUV stellar radiation (Bowyer 1991; Seon et al. 2011).

Therefore, comparison of the diffuse $\mathrm{H} \alpha$ emission with the FUV continuum background could not only provide a clue to the spectral type of ionizing sources of the WIM in our Galaxy but also confirm the significance of dust-scattering in the diffuse $\mathrm{H} \alpha$. In fact, Seon et al. (2011) found a strong correlation between the diffuse FUV (1370-1710 $)$ and $\mathrm{H} \alpha$ backgrounds, suggesting a similarity between the origins of both backgrounds. In this paper, we present a more detailed comparison of the Galactic $\mathrm{H} \alpha$ map with the FUV continuum map and propose a scenario on the origin of the diffuse $\mathrm{H} \alpha$ emission. We describe the data used in the present study in Section 2. In Section 3, the spectral type of the stars responsible for the diffuse $\mathrm{H} \alpha$ emission is investigated. Section 4 contains a 
discussion on the dust-scattering of $\mathrm{H} \alpha$ photons. We discuss the main result in Section 5. A brief summary is presented in Section 6.

\section{DATA}

The FUV data was obtained by the Spectroscopy of Plasma Evolution from Astrophysical Radiation (SPEAR) instruments, also known as Far Ultraviolet Imaging Spectrograph (FIMS) (Edelstein et al. 2006a,b; Seon et al. 2011). The SPEAR/FIMS instrument is a dual-channel FUV imaging spectrograph ("Short" wavelength channel [S-band] 900$1150 \AA$, "Long" wavelength channel [L-band] 1350-1750 $\lambda / \Delta \lambda \sim 550$ ) with a large field of view (S-band, $4^{\circ} \times 4^{\prime} .6$; L-band, $7^{\circ} .4 \times 4^{\prime} .3$ ) and $10^{\prime}$ imaging resolution onboard the first Korean astronomical satellite STSAT-1, designed to observe diffuse FUV emission. The SPEAR/FIMS survey observations were performed by scanning the sky at constant ecliptic longitude from the north ecliptic pole to the south ecliptic pole, during the eclipses. The pole-to-pole scanning drifted $360^{\circ}$ along the ecliptic equator for one year because of the properties of a sun-synchronous orbit. The mission observed $\sim 80 \%$ of the sky.

The diffuse FUV map in L-band was obtained by eliminating stars from the total FUV sky map and by binning photon events using the Hierarchical Equal Area isoLatitude Pixelization (HEALPix) tessellation scheme (Górski et al. 2005) with $\sim 1^{\circ}$ pixels (resolution parameter $N_{\text {side }}=64$ ). We excluded the wavelength regions of the strongest emission lines (Si IV $\lambda 1398, \mathrm{Si}$ II* $\lambda 1532, \mathrm{C}$ IV $\lambda \lambda 1548,1551$, and Al II $\lambda 1671)$ and averaged the data in the wavelength regions of $1370-1520 \AA, 1560-1660 \AA$, and $1680-1710 \AA$. A detailed description of the FUV continuum map is presented in Seon et al. (2011).

The all-sky survey maps in $\mathrm{H} \alpha$ and $100 \mu \mathrm{m}$ were obtained from Finkbeiner (2003) and Schlegel et al. (1998), respectively. The all-sky map of $\mathrm{H}$ I column density is from the Leiden/Argentine/Bonn (LAB) Survey (Kalberla et al. 2005). The data interpolated onto a HEALPix projection were obtained from the Legacy Archive for Microwave Background Data Analysis (LAMBDA).

\section{SPECTRAL TYPE OF IONIZING SOURCES}

Figure 1 shows the $\mathrm{H} \alpha / \mathrm{FUV}$ intensity ratio maps obtained by dividing the $\mathrm{H} \alpha$ intensity with the FUV continuum intensity and smoothed with a spherical Gaussian function with a full-width at half maximum (FWHM) $\sim 3^{\circ}$ (Seon 2006). The smoothing was performed using the spherical harmonic transform in the HEALPix scheme so to smear the same surface areas in all directions. We excluded the regions with low signal-to-noise ratio $(\mathrm{S} / \mathrm{N}<3)$ and two regions between edges of observing strips centered at $(l, b) \sim\left(45^{\circ},-35^{\circ}\right)$ and $\sim\left(225^{\circ}, 45^{\circ}\right)$, which appeared to produce an artifact with very high $\mathrm{H} \alpha / \mathrm{FUV}$ intensity ratios after the smoothing. The FUV and $\mathrm{H} \alpha$ intensities are expressed in units of rayleighs (1 rayleigh $[\mathrm{R}]=10^{6} / 4 \pi$ photons $\mathrm{cm}^{-2} \mathrm{~s}^{-1} \mathrm{sr}^{-1}$ ) and continuum unit $\left(1 \mathrm{CU}=\right.$ photons $\left.\mathrm{cm}^{-2} \mathrm{~s}^{-1} \mathrm{sr}^{-1} \AA^{-1}\right)$. In the figure, the region with higher $\mathrm{H} \alpha / \mathrm{FUV}$ ratio is represented in blue. Top and bottom panels show the intensity ratio maps before and after dust-extinction correction, respectively. Using the standard approach of Bennett et al. (2003) for the emitting medium coexisting with dust and the extinction map of Schlegel et al. (1998), the correction for dust-extinction was performed for both radiations. In the top panel of the figure, it is clear that bright $\mathrm{H}$ II regions have higher $\mathrm{H} \alpha / \mathrm{FUV}$ ratios than the diffuse $\mathrm{H} \alpha$ regions. Correction for dust-extinction lowers the ratio, because the correction for FUV is larger than for $\mathrm{H} \alpha$. As dust-extinction is stronger at low latitudes, the correction for H II regions is bigger than for the WIM. Therefore, the distinction between the bright H II and the WIM regions becomes less clear after the dust-extinction correction, as shown in the bottom panel.

For the quantitative comparison, the WIM regions (we will use the term WIM to denote the diffuse $\mathrm{H} \alpha$ regions that are not related to the bright $\mathrm{H}$ II regions, regardless of physical origin) were isolated in the $\mathrm{H} \alpha$ image using a masking procedure similar to that described in Hoopes et al. (2001). The $\mathrm{H} \alpha$ image was smoothed with a median filter using a pixel size of $\sim 15^{\circ}$ which corresponds approximately to the size of the largest H II regions. The smoothed image was subtracted from the original, leaving an image of the small-scale structures only. In other words, the smoothly varying component was subtracted. On this image a mask was created by assigning the value ' 1 ' to the pixels with intensity lower than a prescribed threshold and ' 0 ' to the others. We tried several threshold values between 0.1 and $50 \mathrm{R}$ as the separation between the WIM and H II regions was rather fuzzy. The unsmoothed $\mathrm{H} \alpha$ image was then multiplied by the mask, leaving an image of only the WIM. A WIM FUV image was also created using the same mask. The $\mathrm{H} \alpha$ and FUV images for $\mathrm{H} \mathrm{II}$ regions were created by multiplying the inverted WIM mask.

Figure 2(a) shows the histograms of the H $\alpha / \mathrm{FUV}$ intensity ratios for the WIM and $\mathrm{H}$ II regions in our Galaxy, with the thresholds of 1, 5, 10 and $20 \mathrm{R}$. The threshold ' $1 \mathrm{R}$ ' masks most of the H II regions and ' 20 R' the brightest ones. The mean ratios for the $\mathrm{H}$ II regions are generally higher than the mean ratios in the WIM. The difference in the logarithm of the average ratio between the $\mathrm{H}$ II regions and the WIM increases smoothly from $0.40 \pm 0.40$ to $0.62 \pm 0.39$ dex as the threshold increases from 1 to $20 \mathrm{R}$. These values are smaller than those found in 10 external galaxies in Hoopes et al. (2001), although the largest value of $\sim 0.62$ is consistent with the results for NGC 2903, NGC 5457, and NGC 1512 within the error ranges. Figure 2(b) shows the histograms of the $\mathrm{H} \alpha / \mathrm{FUV}$ intensity ratios after dust-extinction correction. Correction for dust-extinction lowers the difference in the average ratio, as in the bottom panel of Figure 1.

The smaller difference of the mean ratios in our Galaxy than those of other galaxies may be attributable to geometrical effects, as the WIM and H II regions overlap in our Galaxy while in the face-on external galaxies examined in Hoopes \& Walterbos (2000) and Hoopes et al. (2001) two regions are better separated from each other. The field stars in the Galactic plane overlapping with $\mathrm{H}$ II regions would lower the $\mathrm{H} \alpha / \mathrm{FUV}$ intensity ratio observed toward the bright $\mathrm{H}$ II regions. Some of the $\mathrm{H} \alpha$ and FUV photons originating from $\mathrm{H}$ II regions are scattered into high latitudes (Wood \& Reynolds 1999; Witt et al. 2010; Seon et al. 2011). The dust-scattering of $\mathrm{H} \alpha$ and FUV photons from the $\mathrm{H}$ II regions to high latitudes would then increase the ratio for the WIM, further lowering the difference of the ratios. Furthermore, all the $\mathrm{H}$ II regions are at the same distance in a face-on external galaxy while in the Milky Way the apparent size of $\mathrm{H}$ II regions is potentially dominated by their distances making the separation more difficult. Therefore, the difference between the H II regions and the WIM in our Galaxy may not be significantly different from other galaxies when the Galaxy is viewed face-on. 
Along the top axis of Figure 2 are shown the stellar spectral types that would yield the corresponding $\mathrm{H} \alpha / \mathrm{FUV}$ ratios. We derived a temperature and effective gravity for given spectral types using the calibration of Vacca et al. (1996). We then calculated the Lyc and FUV photon luminosities $L_{\mathrm{Lyc}}$ and $L_{\mathrm{FUV}}$ for spectral types of B2-O3 stars in the wavebands $\lambda<912 \AA$ and $1370-1710 \AA$, respectively, by interpolating on a grid of Kurucz models (Castelli \& Kurucz 2003). The $\mathrm{H} \alpha$ luminosities $L_{\mathrm{H} \alpha}$ equal $0.46 L_{\mathrm{Lyc}}$, assuming case B recombination. Since Vacca et al. (1996) covers spectral types only earlier than or equal to B0.5, we extrapolated the calibration down to B2. The temperatures estimated in this way were $\sim 5-19 \%$ higher than the values obtained from the older calibration of Straižys \& Kuriliene (1981). The extraporation to B2 would be accurate within $\sim 19 \%$. Figure 2 suggests that the difference between the H $\alpha / \mathrm{FUV}$ ratios in the WIM and those in the $\mathrm{H}$ II regions is due to the difference in main ionizing sources.

An interesting result in Figure 2 is that the median value of the observed ratios in the WIM corresponds to a value predictable from $\sim \mathrm{O} 9-\mathrm{B} 0$ stars with effective temperatures of $\sim 35,000 \mathrm{~K}$. Sembach et al. (2000) modeled the WIM as a combination of overlapping $\mathrm{H}$ II regions and found that typical values of the line ratios [S II] $\lambda 6716 / \mathrm{H} \alpha \sim 0.2-0.4$, [N II] $\lambda 6583 / \mathrm{H} \alpha \sim 0.3-0.6,[\mathrm{O}$ I] $\lambda 6300 / \mathrm{H} \alpha \sim 0.01-0.03,[\mathrm{O}$ III] $\lambda 5007 / \mathrm{H} \alpha \lesssim 0.1$, and He I $\lambda 5876 / \mathrm{H} \alpha<0.02$ observed in the Galactic WIM can be well reproduced by an ionizing spectrum of a star with $T_{\text {eff }} \sim 35,000 \mathrm{~K}$; Domgörgen \& Mathis (1994) also found that the WIM observations best agree with $T_{\text {eff }} \sim 38,000 \mathrm{~K}$. The results are consistent with the $\mathrm{H} \alpha / \mathrm{FUV}$ ratios, thereby strongly supporting that the $\mathrm{H} \alpha$ emission originates from late OB-type stars. The optical line ratios will be discussed more in Section 5. We also note that the H $\alpha / \mathrm{FUV}$ ratio has a well-defined limiting value corresponding to B2 stars, meaning that the latest stellar type that is capable of ionizing the WIM may be about B2.

The mean $\mathrm{H} \alpha / \mathrm{FUV}$ intensity ratio after and before the dustextinction correction as a function of $\mathrm{H} \alpha$ intensity is shown together with the standard deviation of ratios from the mean value for a given $\mathrm{H} \alpha$ intensity inverval in Figure 3(a). Uncertainty of the ratio toward an individual sightline is much smaller than the deviation of ratios. Red and black symbols represent the intensity ratio before and after the dustextinction correction, respectively. There is a general trend for the average ratio to increase with the $\mathrm{H} \alpha$ intensity, although it scatters a lot. Both the general trend and large scatter are qualitatively similar to those found in the external galaxies in Hoopes et al. (2001). The scatter in the ratio tends to increase with the $\mathrm{H} \alpha$ intensity. At low latitudes and highest $\mathrm{H} \alpha$ intensities, the $\mathrm{H} \alpha$ radiation may be dominated by recombination lines originated from $\mathrm{H}$ II regions around early O-type stars, while the FUV background is from dust-scattering (Seon et al. 2011). Therefore, a much wider range of variability in the $\mathrm{H} \alpha$ intensity and the $\mathrm{H} \alpha / \mathrm{FUV}$ ratio is found. On the other hand, at high latitudes and low values of $\mathrm{H} \alpha$ intensity, much of the structure in the faint $\mathrm{H} \alpha$ background may have the same origin as that of the FUV background and the variation of $\mathrm{H} \alpha / \mathrm{FUV}$ decreases. In other words, the $\mathrm{H} \alpha$ contribution from dust-scattering becomes increasingly important at higher latitudes. A similar feature was found in Figure 21 of Seon et al. (2011), where a linear correlation of the $\mathrm{H} \alpha$ intensity with the FUV intensity was found only in low intensities. In addition, at lowest $\mathrm{H} \alpha$ intensities, only early B-type stars would contribute to both backgrounds and the ratio would then converge to a single value. The converged value corresponds to a spectral type of $\mathrm{B} 0-\mathrm{B} 1$.

Interestingly, line ratios $[\mathrm{S} \mathrm{II}] / \mathrm{H} \alpha$ and $[\mathrm{N}$ II] $] / \mathrm{H} \alpha$, and the $\mathrm{H} \alpha /$ FUV ratio show a similar trend with decreasing $\mathrm{H} \alpha$ intensity, except the line ratios increase while the $\mathrm{H} \alpha / \mathrm{FUV}$ ratio decreases. Mean values of the optical line ratios increase with decreasing $\mathrm{H} \alpha$ intensity. The ratios also show large scatter as the $\mathrm{H} \alpha / \mathrm{FUV}$ ratio does. The mean $\mathrm{H} \alpha / \mathrm{FUV}$ ratio decreases rapidly below $\mathrm{H} \alpha$ intensity of $\sim 0.7-1 \mathrm{R}$ with decreasing $\mathrm{H} \alpha$ intensity in Figure 3(a). The mean line ratios increase most dramatically below the same $\mathrm{H} \alpha$ intensity $(\sim 0.7-1 \mathrm{R})$ (Figures 6, 12, and 15 in Madsen et al. 2006). The similar trend in both the optical line ratios and the $\mathrm{H} \alpha / \mathrm{FUV}$ ratio may be understood as an increasing contribution of late OB stars to the WIM as the $\mathrm{H} \alpha$ intensity decreases.

We also plotted the hardness ratio in FUV wavelengths versus the $\mathrm{H} \alpha / \mathrm{FUV}$ ratio as the FUV hardness ratio might also provide an additional clue on the spectral type of the ionizing source of the WIM. The hardness ratio is defined as the ratio of average intensities at $\lambda \lambda 1370-1520 \AA$ and $\lambda \lambda 1560-1710 \AA$, excluding the bright C IV, Si II, and Al II emission lines, as in Seon et al. (2011). We found an overall correlation between the FUV hardness ratio with the $\mathrm{H} \alpha / \mathrm{FUV}$ intensity ratio, as shown in Figure 3(b), where the standard deviation of the FUV hardness ratios from its mean value for a given $\mathrm{H} \alpha / \mathrm{FUV}$ bin is also indicated. The mean FUV hardness ratio shows rather rapid decrease below the $\mathrm{H} \alpha / \mathrm{FUV} \sim 1$ $\left(\mathrm{R} / 10^{3} \mathrm{CU}\right)$, corresponding to the approximate mean value of the $\mathrm{H} \alpha / \mathrm{FUV}$ ratio of the WIM. Seon et al. (2011) found that a map of the hardness ratio of $1370-1520 \AA$ to $1560-1710 \AA$ band intensity shows that the sky is divided into roughly two parts, which are determined by the longitudinal distribution of OB-type stars in the Galactic plane. Therefore, the correlation between the FUV hardness ratio and a large portion, if not all, of the $\mathrm{H} \alpha$ /FUV ratio supports that the diffuse $\mathrm{H} \alpha$ background is produced by relatively late-type stars rather than H II regions in the Galactic plane.

Statistically significant correlation coefficients of 0.9 and 0.8 for Figure 3(a) and (b), respectively, were obtained. We also note that most of the $\mathrm{H} \alpha / \mathrm{FUV}$ intensity ratios are so concentrated to $\sim 1\left(\mathrm{R} / 10^{3} \mathrm{CU}\right)$, as can be noted in Figure 2, that the two-dimensional histograms and/or contours as in Figure 4 show strong peaks at the H $\alpha / \mathrm{FUV}$ intensity ratio of $\sim 1$ $\left(\mathrm{R} / 10^{3} \mathrm{CU}\right)$. Therefore, the average intensity ratio versus the abscissa in Figure 3 was the best way to represent clearly the correlations. We note that the $\mathrm{H} \alpha / \mathrm{FUV}$ ratio map (Figure 1) does not match perfectly with the FUV hardness ratio map (Figure 14 of Seon et al. 2011). However, the maps generally correlate well with each other, except a few regions such as near $(l, b) \sim\left(30^{\circ}, 30^{\circ}\right)$.

Hoopes \& Walterbos (2000) and Hoopes et al. (2001) compared the observed $\mathrm{H} \alpha / \mathrm{FUV}$ ratios with the theoretical calculations assuming that the FUV photons are emitted from unresolved point sources in the observed regions. However, FUV photons are well scattered by interstellar dust grains to large distances and the diffuse FUV emission is wide-spread over regions where no point sources are present (Bowyer 1991; Witt et al. 1997; Schiminovich et al. 2001; Seon et al. 2011). The FUV intensity used in the analysis of face-on galaxies would be produced by in-situ point sources as well as dustscattered light in the regions, whereas the FUV data in the present study is dominated by dust-scattered light originating 
from late O- and early B-type stars (Henry 2002; Seon et al. 2011). We also note that the spectral type of dominant radiation source of the FUV continuum background is consistent with that inferred from $\mathrm{H} \alpha / \mathrm{FUV}$ intensity ratio.

It would be interesting to estimate how a large portion of the ionizing power required for the $\mathrm{H} \alpha$ background can be provided by late $\mathrm{OB}$ stars. If the $\mathrm{H} \alpha$ emission originates from ionized gas in a plane-parallel geometry, the hydrogen recombination rate $r_{G}$ per $\mathrm{cm}^{2}$ of galactic disk in the vicinity of the Sun is given by

$$
r_{G} \approx 4 \times 10^{6} I_{\mathrm{H} \alpha}(b) \times \sin |b| \mathrm{s}^{-1} \mathrm{~cm}^{-2},
$$

where $I_{\mathrm{H} \alpha}(b)$ is the $\mathrm{H} \alpha$ intensity at galactic latitude $b$ (Reynolds 1984). Using an approximate $\mathrm{H} \alpha$ intensity distribution $I_{\mathrm{H} \alpha}(b) \approx 1.0 \csc |b| \mathrm{R}$, which was obtained from rather limited observations, Reynolds obtained $r_{G} \approx 4 \times 10^{6} \mathrm{~s}^{-1}$ $\mathrm{cm}^{-2}$. However, using the result of Hill et al. (2008) obtained from the full WIM observations, the distribution of $\mathrm{H} \alpha$ at high latitudes is fitted on average by $I_{\mathrm{H} \alpha}(b) \approx 0.625 \mathrm{csc}|b| \mathrm{R}$ (see also Dong \& Draine 2011). This leads to a lower recombination rate of $r_{G} \approx 2.5 \times 10^{6} \mathrm{~s}^{-1} \mathrm{~cm}^{-2}$. The Lyc luminosities per $\mathrm{cm}^{2}$ of the Galactic disk estimated for the solar neighborhood are $(10-29) \times 10^{6}$ and $(0.5-1) \times 10^{6}$ photons s $\mathrm{sm}^{-1}$ for $\mathrm{O}$ and B stars, respectively (Reynolds 1984). The fraction of Lyc luminosity of O9 and O9.5 stars in total Lyc luminosity due to O stars is $\sim 7-10 \%$ (Terzian 1974; Torres-Peimbert et al. 1974). The Lyc luminosity from the stars later than or equal to O9 would then be $(1.2-3.9) \times 10^{6}$ photons s${ }^{-1} \mathrm{~cm}^{-2}$. Therefore, late $\mathrm{OB}$ stars are able to account for at least one-half of the required ionizing power.

In fact, Elmegreen (1975) considered the intensity of the $\mathrm{H} \alpha$ background at $|b|<30^{\circ}$ and concluded that isolated $\mathrm{H} \mathrm{II}$ regions surrounding early $\mathrm{B}$ stars could account for up to onehalf of the observed $\mathrm{H} \alpha$ background in some regions of the Galactic plane. He predicted that near the Galactic poles, B star $\mathrm{H}$ II regions would account for a mean $\mathrm{H} \alpha$ intensity of $\sim 0.1 \mathrm{R}$. This result is consistent with our estimation.

\section{DUST SCATTERING}

The late OB-type stars may not have enough power to produce the whole $\mathrm{H} \alpha$ background if the $\mathrm{H} \alpha$ photons originate purely from ionized gas around late OB stars, as noted by Reynolds (1984) and in Section 3. However, if dust-scattering plays an important role, the dust-scattered $\mathrm{H} \alpha$ photons originating from the sources other than $\mathrm{O}$ stars in bright $\mathrm{H}$ II regions would contribute a larger portion to the $\mathrm{H} \alpha$ background at high-latitudes and the severe requirement of Lyc leakage from $\mathrm{H}$ II regions in the midplane may be potentially alleviated. In this section, we examine how the contribution of dust-scattering could be significant in the $\mathrm{H} \alpha$ background.

Galactic observables may generally correlate with each other because of the general property of a plane-parallel ISM. The correlation between a pair of Galactic quantities would be enhanced when their sources and radiative transfer mechanisms are analogous. If a large portion of the diffuse $\mathrm{H} \alpha$ intensity is due to dust-scattering, the $\mathrm{H} \alpha$ intensity would show a better correlation with FUV intensity than that with other quantities, such as neutral hydrogen column density $N(\mathrm{H} \mathrm{I})$. On the other hand, if the radiative transfer mechanism for the diffuse $\mathrm{H} \alpha$ emission is not related with the dust-scattering, the correlation between the $\mathrm{H} \alpha$ and FUV intensities would not be improved.

Figure 4 shows the correlation plots of $\mathrm{H} \alpha$ intensity with
$100 \mu \mathrm{m}$ dust emission and $\mathrm{H}$ I column density $N(\mathrm{H} \mathrm{I})$ in the same forms of two-dimensional histograms and contours as in Figure 21 of Seon et al. (2011), in which the FUV intensity is compared with $100 \mu \mathrm{m}$ intensity, $N(\mathrm{H} \mathrm{I})$, and $\mathrm{H} \alpha$ intensity. Correlation coefficients estimated in logarithmic scale are also shown in the figure. We find that the correlation of $\mathrm{H} \alpha$ intensity with FUV (shown in Figure 21(c) of Seon et al. 2011) is a bit better, at least qualitatively, than with the others ISM tracers shown in Figure 4. The correlation relations with 100 $\mu \mathrm{m}$ intensity and $N(\mathrm{H} \mathrm{I})$ are a bit curved and flaring, whereas the correlation with the FUV background is quite straight. However, the correlation coefficient between the $\mathrm{H} \alpha$ and FUV backgrounds is not significantly higher than the other correlation coefficients.

The fact that the correlation coefficients show no significant differences may be caused by large scatter in the correlation relations and the general similarity in the latitude dependence of the data sets (the H I column density, $100 \mu \mathrm{m}$, $\mathrm{H} \alpha$, and FUV intensities). Although the $\mathrm{H} \alpha$ emissivity and $\mathrm{H}$ I column density have different dependences on the gas density, their latitude dependences have the same trend with inverse-sin $|b|$ if they arise in plane-parallel geometries. The inverse-sin $|b|$ dependence of the data sets dominates the correlations in Figure 4. To reduce the latitude dependence and scatter, we therefore plotted the average intensities multiplied by $\sin |b|$ versus $\sin |b|$ within each of the $\Delta \sin |b|=0.01$ latitude intervals for $N(\mathrm{H} \mathrm{I}), 100 \mu \mathrm{m}, \mathrm{H} \alpha$, and FUV intensities in Figure 5. In the figure, the curves were paired and arbitrarily moved to compare the shapes. The top two curves compare the $N(\mathrm{H} \mathrm{I})$ and $100 \mu \mathrm{m}$ emission. The middle and bottom pairs show the variations of $\mathrm{H} \alpha$ and FUV intensities after and before dust-extinction correction, respectively. The $N(\mathrm{H} \mathrm{I})$ and $100 \mu \mathrm{m}$ emission are well described by the planeparallel or inverse-sin $|b|$ law, whereas $\mathrm{H} \alpha$ and FUV intensities deviate from the law. The deviation of $\mathrm{H} \alpha$ and FUV intensities from the inverse-sin $|b|$ law is mainly attributable to the effect of dust absorption and scattering. A radiative transfer model of diffuse Galactic light in plane-parallel dust layer shows the similar dependence of $I \sin |b|$ on $\sin |b|$ as in the bottom curves of Figure 5 (van de Hulst \& de Jong 1969; see also Wood \& Reynolds 1999 and Seon 2009b). We also note that the inverse-sin $|b|$ equation of the $\mathrm{H} \alpha$ intensity described in Section 3 is still a good approximation because of the major dependence of the $\mathrm{H} \alpha$ intensity on inverse-sin $|b|$, especially at high latitudes, even $I \sin |b|$ is not a constant in Figure 5.

We note from the bottom two curves before dust-extinction correction that the dependences of the $\mathrm{H} \alpha$ and FUV backgrounds on the Galactic latitude are very similar at high latitudes $(\sin |b|>0.3)$ (See also Figure 13 of Haffner et al. 1999 and Figure 9 of Seon et al. 2011), whereas at low latitudes $(\sin |b|<0.3)$ they show a difference. Stronger dustextinction in FUV would suppress the FUV intensity near the Galactic plane, as shown in low latitudes. We therefore corrected the dust-extinction as denoted by the middle two curves (red and black solid lines for $\mathrm{H} \alpha$ and FUV, respectively). The dust-extinction correction for both backgrounds was performed using the standard approach of Bennett et al. (2003). The variations of both backgrounds are remarkably similar to each other. The FUV radiation can be emitted even from late B-type stars, whereas the $\mathrm{H} \alpha$ emission requires excitation from earlier stars. Therefore, the scaleheight of the FUV sources is a bit higher than that of the $\mathrm{H} \alpha$ sources. On the other hands, ionized $\mathrm{H}$ II regions must be more extended than point sources for the FUV photons, and the dust-scattered 
$\mathrm{H} \alpha$ originating from $\mathrm{H}$ II regions should be more extended than those from point sources. Accordingly, the dependences of both backgrounds on the Galactic latitude are very similar even at low latitudes, after extinction corrections are applied.

In Figure 6, we also examine correlations of the diffuse $\mathrm{H} \alpha$ intensity with $N(\mathrm{H} \mathrm{I}), 100 \mu \mathrm{m}$ emission, and the diffuse FUV background after the removal of inverse-sin $|b|$ dependence. In the correlation plot, we used only the latitude range of $\sin |b|>0.3$ to avoid the strong dust-extinction effect. Red and black symbols represent the data points after and before the dust-extinction correction, respectively. The figure also shows correlation coefficients. It is obvious that the correlation with the FUV background is now significantly stronger than the correlations with others. In Seon et al. (2011), we compared the FUV continuum background with other ISM tracers and found that the FUV intensity correlates better with the $\mathrm{H} \alpha$ intensity than with others. The two-photon emission in the WIM is unlikely to be the cause of the strong correlation between the $\mathrm{H} \alpha$ and FUV backgrounds (Seon et al. 2011). We are therefore convinced of the result that a significant portion of the diffuse $\mathrm{H} \alpha$ emission at high latitudes is dust-scattered light originated from elsewhere in the Galaxy.

We now estimate the fraction of dust-scattered $\mathrm{H} \alpha$ emission. Seon et al. (2011) derived two equations (Equations (7) and (8)) relating the FUV intensity to $100 \mu \mathrm{m}$ and $\mathrm{H} \alpha$ emissions at high latitudes. Since the constant terms in the relations are often regarded as extragalactic background, they should be identical. The difference between the constant terms is indeed negligible. Therefore, the relation between the $100 \mu \mathrm{m}$ and $\mathrm{H} \alpha$ emission are given by $I_{100 \mu \mathrm{m}} \approx 2.88\left(I_{\mathrm{H} \alpha} / R\right)$ $\mathrm{MJy} / \mathrm{sr}$. Witt et al. (2010) provides an empirical relation, which relates the scattered $\mathrm{H} \alpha$ intensity to the $100 \mu \mathrm{m}$ emission at high latitudes. Using these relations, we obtain an average ratio of the scattered $\mathrm{H} \alpha$ intensity to total intensity, $I_{\mathrm{H \alpha}}^{\text {scatt }} \approx 0.37 I_{\mathrm{H} \alpha}$. Therefore, in general, about $37 \%$ of the diffuse $\mathrm{H} \alpha$ emission at high latitudes would be due to dustscattering. Contribution of the Lyc leakage from bright H II regions may be $\sim 13 \%$ on average if late OB stars produce about half of the $\mathrm{H} \alpha$ emission. However, these numbers should be referenced with caution. The scattered portion can be much higher or lower depending on location, as shown in Witt et al. (2010). Since the FUV background traces the dustscattered light, we expect that the FUV to $\mathrm{H} \alpha$ ratio would be more or less proportional to the scattered fraction of $\mathrm{H} \alpha$ background. We note that the scattered fraction of $\mathrm{H} \alpha$ background shown in Figure 5 of Witt et al. (2010) is more or less anticorrelated with the $\mathrm{H} \alpha / \mathrm{FUV}$ ratio map of Figure 1, verifying the expectation.

\section{DISCUSSION}

By combing the previous results, we propose that most of the diffuse $\mathrm{H} \alpha$ background at high latitudes may originate from relatively late $\mathrm{O}$ - and early $\mathrm{B}$-stars outside the bright $\mathrm{H}$ II regions and from dust-scattered $\mathrm{H} \alpha$ photons from elsewhere in the Milky Way. The observed H $\alpha /$ FUV intensity ratio in general increases with the $\mathrm{H} \alpha$ intensity, indicating that late $\mathrm{OB}$ stars could be important contributors to the diffuse $\mathrm{H} \alpha$. As suggested by the strong correlation between the diffuse $\mathrm{H} \alpha$ and FUV backgrounds, dust-scattering may widely spread out the $\mathrm{H} \alpha$ recombination photons into regions where there is no ionizing source.

\subsection{Ionizing and heating sources}

The present scenario is able to explain the line ratios observed in the WIM much more easily than or equally as well as the photoionization models. The higher [S II] $\lambda 6716 / \mathrm{H} \alpha$ and [N II] $\lambda 6583 / \mathrm{H} \alpha$ line ratios observed in the diffuse $\mathrm{H} \alpha$ regions compared to those in $\mathrm{H}$ II regions have been believed to be the strongest evidence against the theory of a dust-scattering origin of the diffuse $\mathrm{H} \alpha$ emission (Reynolds 1990b). However, we have to note that the argument has been based solely on comparison with the line ratios from the bright $\mathrm{H}$ II regions. If the line ratios from the ionized regions due to the late-type $\mathrm{OB}$ stars are compared with the line ratios in the WIM, the present scenario seems to explain the trend equally as well as the previous photoionization models do.

As already noted in Section 3, mean values of [S II]/H $\alpha$ and $[\mathrm{N} \mathrm{II}] / \mathrm{H} \alpha$ intensity ratios in the diffuse $\mathrm{H} \alpha$ regions are well reproduced with late OB stars (Sembach et al. 2000). Figure 3 in Reynolds (1988) shows that the [S II]/H $\alpha$ ratios observed in $\mathrm{H}$ II regions around late-type stars are higher than those found around earlier-type stars. Recently, O'Dell et al. (2011) pointed out that the similarity of the line ratios between the Barnard's Loop, the Orion-Eridanus Bubble, and the typical WIM samples is striking and probably indicates a common set of their physical conditions. The ionizing stars of the Barnard's Loop are O9.5 and O9 types. They found that the photoionization models with the ionization parameter $\log U=$ $-3.07 \sim-3.67$ and the stellar temperature $T_{\text {star }}=31,000 \mathrm{~K}-$ $40,000 \mathrm{~K}$ fully enclose the space of $[\mathrm{S} \mathrm{II}] / \mathrm{H} \alpha$ versus $[\mathrm{N} \mathrm{II}] / \mathrm{H} \alpha$ occupied by Barnard's Loop, the Orion-Eridaunus Bubble, and the WIM samples. It is also noticeable that most of the WIM samples in their Figure 7 occupies the region defined by a stellar temperature lower than 40,000 K, probably $\sim 35,000$ $\mathrm{K}$. Another interesting fact to note is that the elemental abundances required to explain the line ratios in the WIM are close to the B star abundances both in Sembach et al. (2000) and in O'Dell et al. (2011). These results strongly support the late OB star origin of the diffuse $\mathrm{H} \alpha$ emission.

Weak [O III] $\lambda 5007 / \mathrm{H} \alpha$ and $\mathrm{He}$ I $\lambda 5876 / \mathrm{H} \alpha$ emission line ratios also indicate that the spectrum of the diffuse interstellar radiation field is significantly softer than that from the average Galactic O star population (Reynolds \& Tufte 1995; Madsen et al. 2006).

The increase of the line ratios $[\mathrm{S} \mathrm{II}] / \mathrm{H} \alpha$ and $[\mathrm{N} \mathrm{II}] / \mathrm{H} \alpha$ can be understood as a significant ioninzation contribution by late OB stars to the WIM. However, their highest values observed in the WIM may need other non-ionizing heating sources, such as shocks, photoelectric heating, and/or turbulent mixing layers (Reynolds \& Cox 1992; Slavin et al. 1993; Collins \& Rand 2001). Other heating sources may include cooling, falling galactic fountain gas initially raised from the midplane by supernovae and microflares from magnetic reconnection (Raymond 1992; Shapiro \& Benjamin 1993). It should be noted that the pure photoionization models by $\mathrm{O}$ type stars in the midplane also need additional heating sources to explain the highest ratios (Reynolds et al. 1999). The emission lines due to the heating sources that provide the highest line ratios would also be scattered into more extended regions than those originally produced by the heating/ionizing sources. Therefore, the dust-scattering will hamper the clear identification of the original ionization sources.

As noted by Hoopes \& Walterbos (2003), the [S II]/[N II] ratio is almost independent of ionizing stellar temperature but depends on the ionization parameter. The photoionization models explain the enhanced $[\mathrm{S} \mathrm{II}] / \mathrm{H} \alpha$ line ratios by a diluted radiation field and thus the lowered ionization param- 
eter. Hence the models cannot reproduce the observed constancy of the $[\mathrm{S} \mathrm{II}] /[\mathrm{N} \mathrm{II}]$ ratios. Instead, the dust-scattering scenario can naturally explain the constancy of the line ratios.

\subsection{Physical properties}

Dust scattering has a significant impact on line ratios which are used to determine physical properties of $\mathrm{H}$ II regions (O'Dell \& Goss 2009; O’Dell \& Harris 2010). Scattered light is enhanced at shorter wavelengths, which can lead to observed Balmer line ratios that are theoretically impossible. This effect can also lead to overestimates of the electron temperatures derived from the auroral and nebular line ratios of forbidden lines, such as [N II] $\lambda 5755 /[\mathrm{N} \mathrm{II]} \lambda 6583$ which has been used to derive the temperature of the WIM. Serious discrepancies between the $\mathrm{H} \alpha$ intensity and radio and/or IR intensities could be found when a large portion of the $\mathrm{H} \alpha$ intensity is due to dust-scattering. This effect was found in the Extended Orion Nebula (EON). O'Dell \& Goss (2009) combined $327.5 \mathrm{MHz}$ radio observations and optical spectroscopy to study conditions in the EON. They found an increase in the ratio of emission measures derived from the $\mathrm{H} \beta$ line and the $327.5 \mathrm{MHz}$ radio continuum with increasing distance from the dominant photoionizing star $\theta^{1}$ Ori $\mathrm{C}$, indicating the increasing contribution of a dust-scattering component to the $\mathrm{H} \beta$ intensity with the distance.

Recent observations using the Wilkinson Microwave Anisotropy Probe have found that the ratio of the free-free radio continuum to $\mathrm{H} \alpha$ is surprisingly low in the WIM (Davies et al. 2006; Dobler \& Finkbeiner 2008; Dobler et al. 2009; Gold et al. 2011). Dong \& Draine (2011) proposed a three-component model consisting of a mix of (1) hot gas currently being photoionized, (2) gas that is recombining and cooling after removal of a photoionizing source, and (3) cold $\mathrm{H}$ I gas. In the standard model for explaining the observed intensity ratios of the free-free radio continuum to $\mathrm{H} \alpha$ and [N II] $\lambda 6583$ to $\mathrm{H} \alpha$, they assumed that the scattered fraction of the $\mathrm{H} \alpha$ originating from the hot gas is $20 \%$. The fractions of the hot, cooling, and $\mathrm{H}$ I gases were found to be 22 , 56 , and $2 \%$, respectively. The model predicted that the photoionization should switch off and the gas begins to cool and recombine in a much shorter time $\left(1 \times 10^{5} \mathrm{yr}\right)$ than $\mathrm{O}$ star lifetimes $\left(\sim 3 \times 10^{6} \mathrm{yr}\right)$. Such a short photoionization time scale is rather surprising in that $\mathrm{O}$ stars have been generally favored as the source of ionization for the WIM. They therefore suggested that the ionizing radiation for the WIM may be provided in large part by runaway $\mathrm{O}$ and $\mathrm{B}$ stars, with space velocities $\gtrsim 100 \mathrm{~km} \mathrm{~s}^{-1}$. In the analysis, they assumed the $[\mathrm{N} \mathrm{II}] / \mathrm{H} \alpha$ line ratio of $\sim 0.4$ for the WIM, which is well reproduced by late OB-type stars (Sembach et al. 2000). The ratio of free-free to $\mathrm{H} \alpha$ is estimated to be $\psi=0.16 \mathrm{kJy} \mathrm{sr}^{-1}$ $\mathrm{R}^{-1}$ for the WIM with a temperature of $8000 \mathrm{~K}$ (Equation (11) in Dong \& Draine 2011), which is about 1.9 times larger than the observed ratio of $0.085 \mathrm{kJy} \mathrm{sr}^{-1} \mathrm{R}^{-1}$. If we assume that $\sim 37 \%$ of the observed $\mathrm{H} \alpha$ intensity is from dust-scattering and the temperature of the WIM is $8000 \mathrm{~K}$, the fractions of the hot and cooling gases are found to be $\sim 44 \%$ and $\sim 18 \%$, respectively.

The WIM is thought to have a temperature $2000 \mathrm{~K}$ higher than the bright classical H II regions (Reynolds et al. 2001; Madsen et al. 2006). However, O’Dell et al. (2011) found no evidence that the WIM components have a systematically higher temperature than the well known higher-density $\mathrm{H}$ II regions. As already noted, the temperature derived from
[N II] $\lambda 5755 /[\mathrm{N} \mathrm{II]} \lambda 6583$ may be uncertain. Temperature of the Barnard's Loop is found to be $\sim 5960-6100 \mathrm{~K}$ (Heiles et al. 2000; O'Dell et al. 2011). From the similarity of the physical conditions between the Barnard's Loop and the typical WIM sample, we may assume an extreme case that the temperature of the WIM is $\sim 6100 \mathrm{~K}$, and there is no contribution from either cooling or cold $\mathrm{H}$ I gas. The assumptions may be rather extreme, but still plausible at some sightlines since the physical conditions and constribution of dust scattering in individual sightlines would generally differ from the average and vary from sightline to sightline (Madsen et al. 2006; Witt et al. 2010). The free-free to $\mathrm{H} \alpha$ ratio of $(1-0.37) \psi(6100 \mathrm{~K}) \sim 0.085$ is then obtained, which agrees with the observed ratio. Therefore, relatively late $\mathrm{O}$ and/or early B-type stars, as proposed in the present study, appear to explain the observed values of not only optical line ratios but also free-free to $\mathrm{H} \alpha$ intensity ratios even without considering a significant contribution of either cooling or cold $\mathrm{H} \mathrm{I}$ gas. However, we emphasize that the aim of this study is to explain averaged observational results and our results do not indicate that all the diffuse $\mathrm{H} \alpha$ emission is produced by late $\mathrm{OB}$ stars outside bright $\mathrm{H}$ II regions and dust scattering. Large scatter found in Figure 3 also indicates that not only the physical conditions and contributions of various components (dust-scattered component, hot and cooling gases) but also the ionizing and/or heating sources of the WIM vary significantly from sightline to sightline.

Low extinction at $2.17 \mu \mathrm{m} \mathrm{Br} \gamma$ emission line should allow to probe the WIM throughout the Galactic plane. A pilot survey of the $\mathrm{Br} \gamma$ emission carried out using the GoddardWisconsin near-IR cryogenic spectrometer revealed that the volume filling fraction of the $\mathrm{Br} \gamma$-emitting medium is typically around $1 \%$, indicating the $\mathrm{Br} \gamma$ emission is likely related to more compact sources (Kutyrev et al. 2001, 2004). If the Lyc photons are indeed leaked out of the bright $\mathrm{H}$ II regions in the Galactic plane and photoionize the surrounding medium to produce the diffuse $\mathrm{H} \alpha$ emission, as usually supposed, the filling fraction of the $\mathrm{Br} \gamma$-emitting gas in the Galactic plane should be equal to or higher than the filling fraction of $\sim 10 \%$ obtained by the $\mathrm{H} \alpha$ observations (Kulkarni \& Heiles 1987; Reynolds 1991; Peterson \& Webber 2002). This discrepancy strongly suggests that the Lyc leaked out of bright H II regions is unlikely the major source of the diffuse $\mathrm{H} \alpha$ background and a significant portion of the $\mathrm{H} \alpha$ background originates from dust-scattering. More detailed observations using hydrogen recombination lines in the near-IR wavelenths, such as $\mathrm{Pa} \alpha$ (1.87 $\mu \mathrm{m}), \operatorname{Br} \alpha(4.05 \mu \mathrm{m})$, and $\operatorname{Br} \gamma$, would be needed to understand the origin of the diffuse $\mathrm{H} \alpha$ emission. The Multipurpose Infra-Red Imaging System (MIRIS) is being developed to survey the Galactic plane at $\mathrm{Pa} \alpha$ emission line, which may help to understand the origin of the diffuse $\mathrm{H} \alpha$ emission (Han et al. 2010).

The volume filling fraction and scaleheight of the WIM were estimated from the assumption that the pulsar dispersion measure and the $\mathrm{H} \alpha$ photons probe the same ionized medium. However, as a large portion of the diffuse $\mathrm{H} \alpha$ background at high latitudes may be the result of dust-scattering, the filling fraction and scaleheight must be reexamined. We also found that some of the small-scale features in the FUV sky coincided with the $\mathrm{H} \alpha$ sky, but not always. A detailed examination of small-scale correlation between the $\mathrm{H} \alpha$ and FUV background, which should provide better understanding of the origin of the diffuse $\mathrm{H} \alpha$ emission, will be presented in the future. 


\subsection{Radiative transfer mechanisms}

Assuming the conventional photoionization models of the $\mathrm{H} \alpha$ background, the ISM may have low-density paths and voids that allow for ionizing photons from midplane OB stars to reach and ionize gas many kiloparsecs above the Galactic plane (Wood et al. 2010). Pathways that provide lower than average densities to high latitudes could enhance both scattered FUV and gas ionized by the Lyc from the same OB stars in the Galactic plane. Stars that can reproduce most of the FUV continuum background in the radiative transfer models (e.g., Witt et al. 1997; Schiminovich et al. 2001) are located within only a few hundred pc from the Sun. FUV photons are predominantly scattered in the forward direction (Witt et al. 1997; Schiminovich et al. 2001; Lee et al. 2008), implying that dust-scattering occurs mostly in between the observer and the source. Preliminary results from our MonteCarlo 3D radiative transfer models for the FUV continuum background indicate that most of the FUV continuum background originates from stars and dust located within a volume with a size of $\pm 1 \mathrm{kpc}$ centered at the Sun (Seon et al., in preparation). The $\mathrm{H} \alpha$ photons of the WIM are thought to originate within 2-3 kpc distance (e.g., Reynolds 1984), implying that Lyc photons would also originate from a volume within a few kpc. In this regard, we note that the dust-scattering cross-section at $1550 \AA$ is $\sim 1.3 \times 10^{4}$ times lower than the photoionization cross-section at $912 \AA$ (Weingartner \& Draine 2001; Verner \& Yakovlev 1995), assuming a Galactic dustto-gas ratio. This suggests that FUV photons should originate from much (probably $\sim 10^{4}$ times) larger volume than $\mathrm{H} \alpha$ photons, which is in contrast with the radiative transfer models of the FUV continuum background. Meanwhile, the dust-scattering cross-section for $\mathrm{H} \alpha$ photons is lower than that for FUV $(1550 \AA$ ) by a factor of $\sim 2$ (Weingartner \& Draine 2001), indicating that dust-scattering of the FUV photons will occur at closer distances than $\mathrm{H} \alpha$ photons. Therefore, the dust-scattering of $\mathrm{H} \alpha$ photons provide a much easier way to explain the diffuse $\mathrm{H} \alpha$ background than the conventional photoionization models.

Wood \& Reynolds (1999) found that the $\mathrm{H} \alpha$ intensity from midplane $\mathrm{H}$ II regions that is scattered by dust at high latitudes is in the range $5-20 \%$ of the total intensity. However, we should note that in-situ $\mathrm{H} \alpha$ recombination photons at high latitudes would be also scattered and this component was not included in the estimation. In Figure 3 of Wood \& Reynolds (1999), the scattered $\mathrm{H} \alpha$ intensity that is originating from the WIM through in-situ recombination is compatible with the scattered light from bright H II regions (point sources) at some high latitudes, indicating higher fraction of dust scattering than 5-20\%. Moreover, as noted in Witt et al. (2010), the highly structured distribution in the ratio of $\mathrm{H} \alpha$ scattering to $\mathrm{H} \alpha$ in situ emission was not accounted for in the model of Wood \& Reynolds (1999). The dust-scattered light of in-situ $\mathrm{H} \alpha$ emission (originating mostly from late OB-type stars) at high latitudes, and the complex structures of the ISM and ionizing sources may result in a higher fraction of dust-scattering than predicted by the simplistic calculation.

If late OB stars produce about half of the diffuse $\mathrm{H} \alpha$ intensity, the dust-scattered fraction of the $\mathrm{H} \alpha$ intensity is more or less at the same level as that due to in-situ recombination. The direct FUV starlight and the diffuse FUV background were found to contribute approximately the same amounts to the total FUV intensity on average (Seon et al. 2011). The similarity of the dust-scattered fractions between the FUV con- tinuum and $\mathrm{H} \alpha$ backgrounds supports again the idea wherein their sources and radiative transfer mechanisms are similar.

\section{SUMMARY}

In summary, we found that the $\mathrm{H} \alpha / \mathrm{FUV}$ intensity ratio increases with the $\mathrm{H} \alpha$ intensity and FUV hardness ratio, implying that late OB-type stars mostly produce the diffuse $\mathrm{H} \alpha$ at high latitudes. We also found that the $\mathrm{H} \alpha$ correlates with the FUV background very well. The correlations strongly suggest that a large portion of the diffuse $\mathrm{H} \alpha$ photons originate from late OB-type stars and dust-scattering. We therefore propose a scenario wherein both late OB-type stars and dust-scattering play significant roles in the diffuse $\mathrm{H} \alpha$ emission. The $\mathrm{H} \alpha$ background at high Galactic latitudes may mostly originate from late OB stars existing outside the bright $\mathrm{H}$ II regions and dust-scattering of the $\mathrm{H} \alpha$ photons. The proposed scenario appears to reproduce most of the observed line ratios, such as average values of $[\mathrm{S} \mathrm{III}] / \mathrm{H} \alpha,[\mathrm{N}$ II $] / \mathrm{H} \alpha$, [O III] $/ \mathrm{H} \alpha$ and $\mathrm{He} \mathrm{I} / \mathrm{H} \alpha$, and free-free to $\mathrm{H} \alpha$ ratios. Their extreme values may need additional heating sources, as is also needed in the pure photoionization models. Our scenario is also in better accord with the relative constancy of the $[\mathrm{S} \mathrm{II}] /[\mathrm{N}$ II] intensity ratio, which cannot be explained with the standard photoionization models.

The SPEAR/FIMS is supported by NASA grant NAG55355 and flies on the STSAT-1 Mission, supported of the Korea Ministry of Science and Technology. We acknowledge the use of the Legacy Archive for Microwave Background Data Analysis (LAMBDA). Support for LAMBDA is provided by the NASA Office of Space Science. K.-I. S. was supported by a National Research Foundation of Korea grant funded by the Korean government.

\section{REFERENCES}

Bennett, C. L., Hill, R. S., Hinshaw, G., et al. 2003, ApJS, 148, 97 Bowyer, S. 1991, ARA\&A, 29, 59

Castelli, F., \& Kurucz, R. L. 2003, in IAU Symp. 210 (Cambridge: Cambridge Univ. Press), 20

Collins, J. A., \& Rand, R. J. 2001, ApJ, 551, 57

Davies, R. D., Dickinson, C., Banday, et al. 2006, MNRAS, 370, 1125

Dong, R., \& Draine, B. T., 2011, ApJ, 727, 35

Dove, J. B., \& Shull, J. M. 1994, ApJ, 430, 222

Draine, B. T. 2003, ApJ, 598, 1017

Dobler, G., Draine, B., \& Finkbeiner, D. P. 2009, ApJ, 699, 1374

Dobler, G., \& Finkbeiner, D. P. 2008, ApJ, 680, 1235

Domgörgen, H., \& Mathis, J. S. 1994, ApJ, 428, 647

Edelstein, J., Min, K.-W., Han, W., et al. 2006a, ApJ, 644, L153

Edelstein, J., Korpela, E. J., Adolfo, J., et al. 2006b, ApJ, 644, L159

Elmegreen, B. G. 1975, ApJ, 198, L31.

Ferguson, A. M. N., Wyse, R. F. G., Gallagher, J. S. III, Hunter, D. A. 1996,

AJ, 111, 2265

Finkbeiner, D. P. 2003, ApJS, 146, 407

Gold, B., Odegard, N., Weiland, J. L., et al. 2011, ApJS, 192, 15

Górski, K. M., Hivon, E., Banday, A. J., et al. 2005, ApJ, 622, 759

Han, W., Lee, D.-H., Park, Y., et al. 2010, Proc. of SPIE, 7731, 77311W

Haffner, L. M., Reynolds, R. J., \& Tufte, S. L. 1999, ApJ, 523, 223

Haffner, L. M. 2009, Rev. of Mod. Phys., 81, 696

Heiles, C., Haffner, L. M., Reynolds, R. J., \& Tufte, S. L. 2000, ApJ, 536, 335

Henry, R. C. 2002, ApJ, 570, 697

Hill, A. S., Benjamin, R. A., Kowal, G., et al. 2008, ApJ, 686, 363

Hoopes, C. G., \& Walterbos, R. A. M. 2000, ApJ, 541, 597

Hoopes, C. G., Walterbos, R. A. M., \& Bothun, G. D. 2001, ApJ, 559, 878

Hoopes, C. G., \& Walterbos, R. A. M. 2003, ApJ, 586, 902

Jura, M. 1979, ApJ, 227, 798

Kalberla, P. M. W., Burton, W. B., Hartmann, D., et al. 2005, A\&A, 440, 775 
Kulkarni, S. R., and C. Heiles, 1987, "The atomic component," in Interstellar Processes, edited by D. J. Hollenbach and H. A. Thronson, Jr., Vol. 134 of Astrophysics and Space Science Library (Reidel, Dordrecht, pp. 87-122.

Kutyrev, A. S., Bennett, C. L., Moseley, S. H., Reynolds, R. J., \& Roesler, F. 2001, Tetons 4: Galactic Structure, Stars, \& the Interstellar Medium, eds. C. E. Woodward, M. D. Bicay, and J. M. Shull 360

Kutyrev, A. S., Bennett, C. L., Moseley, S. H., Reynolds, R. J., \& Roesler, F. 2004, How Does the Galaxy Work?, eds. E. J. Alfraro et al. 79

Lee, D.-H., Seon, K.-I., Min, K.-W., et al. 2008, ApJ, 686, 1155

Lehtinen, K., M. Juvela, M., \& Mattila, K. 2010, A\&A, 517, A79

Madsen, G. J., Reynolds, R. J., \& Haffner, L. M. 2006, ApJ, 652, 401

Massey, P., Lang, C. C., DeGioia-Eastwood, K., \& Garmany, C. D. 1995, ApJ, 438, 188

Mathis, J. S. 1986, ApJ, 301, 423

Mattila, K., Juvela, M., \& Lehtinen, K. 2007, ApJ, 654, L131

Miller, W. W., III, \& Cox, D. P. 1993, ApJ, 417, 579

O’Dell, C. R., Ferland, G. J., Porter, R. L., \& van Hoof, P. A. M. 2011, ApJ, 733, 9

O'Dell, C. R., \& Goss, W. M. 2009, AJ, 138, 1235

O’Dell, C. R., \& Harris, J. A. 2010, AJ, 140, 985

Peterson, J. P, \& Webber, W. R. 2002, ApJ, 575, 217

Raymond, J. C. 1992, ApJ, 384, 502

Reynolds, R. J. 1984, ApJ, 282, 191

Reynolds, R. J. 1988, ApJ, 333, 341

Reynolds, R. J. 1990a, ApJ, 349, L17

Reynolds, R. J. 1990b, in IAU Symp. 139, The Galactic and Extragalactic Background Radiation, ed. S. Bowyer \& C. Leinert (Dordrecht : Kluwer), 157

Reynolds, R. J. 1991, ApJ, 372, L17

Reynolds, R. J., \& Cox, D. P. 1992, ApJ, 400, L33

Reynolds, R. J., \& Tufte, S. L. 1995, ApJ, 439, L17
Reynolds, R. J., Hausen, N. R., Tufte, S. L., \& Haffner, L. M. 1998, ApJ, 494, L99

Reynolds, R. J., Haffner, L. M., \& Tufte, S. L. 1999, ApJ, 525, L21

Reynolds, R. J., Sterling, N. C., Haffner, L. M., \& Tufte, S. L. 2001, ApJ, 548, L221

Shapiro, P. R., \& Benjamin, R. A. 1993, in Star Formation, Galaxies, and the Interstellar Medium, ed. J. Franco, F. Ferrini \& G. Tenorio-Tagle (Cambridge Univ. Press), p. 275

Schlegel, D. J., Finkbeiner, D. P., \& Davis, M. 1998, ApJ, 500, 525

Schiminovich, D., Friedman, P. G., Martin, C., \& Morrissey, P. F. 2001, ApJ, 563, L161

Sembach, K. R., Howk, J. C., Ryans, R. S. I., \& Keenan, F. P. 2000, ApJ, 528,310

Seon, K.-I. 2006, J. Korean Phys. Soc., 48, L331 (arXiv:0703168)

Seon, K.-I. 2009a, ApJ, 703, 1159

Seon, K.-I. 2009b, Pub. of Korean Astron. Soc., 24, 1

Seon, K.-I., Edelstein, J., Korpela, E. J., et al. 2011, ApJS, 196, 15

Slavin, J. D., Shull, J. M., \& Begelman, M. C. 1993, ApJ, 407, 83

Straižys, V., \& Kuriliene, G. 1981, Ap\&SS, 80, 353

Terzian, Y. 1974, ApJ, 193, 93

Torres-Peimbert, S., Lazcano-Araujo, A., \& Peimbert, M. 1974, ApJ, 191, 401

Vacca, W. D., Gramany, C. D., \& Shull, J. M. 1996, ApJ, 460, 914

van de Hulst, H. C., \& de Jong, T. 1969, Physica, 41, 151

Verner, D. A., \& Yakovlev, D. G. 1995, A\&AS, 109, 125

Weingartner, J. C., Draine, B. T., 2001, ApJ, 548, 296

Witt, A. N., Friedmann, B. C., \& Sasseen, T. P. 1997, ApJ, 481, 809

Witt, A. N., Gold, B., Barnes, F. S., III, DeRoo, C. T., \& Vijh, U. P. 2010, ApJ, 724, 1151

Wood, K., \& Reynolds, R. J. 1999, ApJ, 525, 799

Wood, K., Hill, A. S., Joung, M. R., et al. 2010, ApJ, 721, 1397 
$\log _{10}\left[\mathrm{H} \alpha / \mathrm{FUV}\left(\mathrm{R} / 10^{3} \mathrm{CU}\right)\right]$

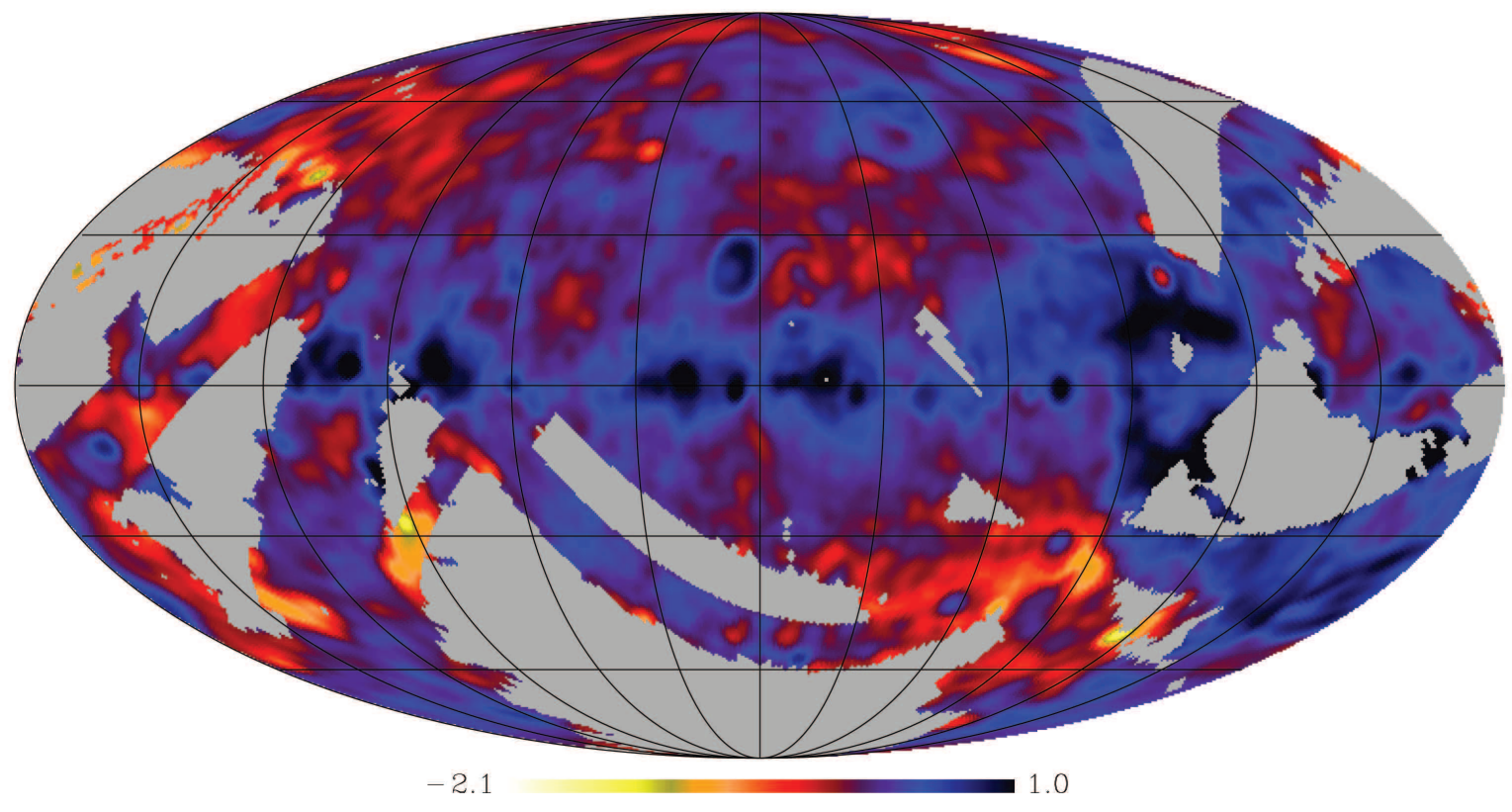

$\log _{10}\left[\mathrm{H} \alpha / \mathrm{FUV}\left(\mathrm{R} / 10^{3} \mathrm{CU}\right)\right]$

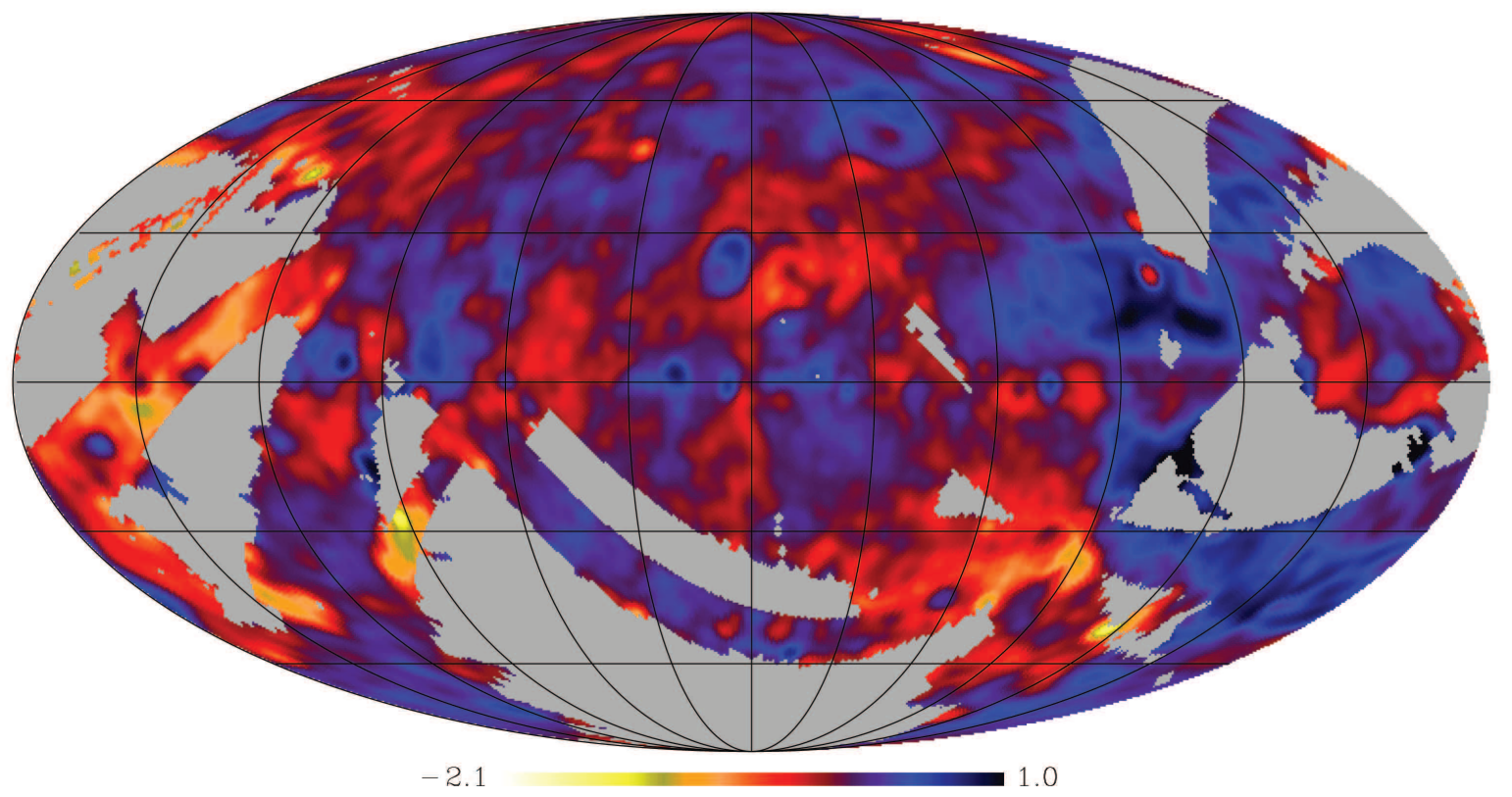

Figure 1. Mollweide projections of the diffuse H $\alpha$ to FUV intensity ratio maps. Top panel shows the ratio map before dust-extinction correction. Bottom panel shows the ratio map after dust-extinction correction. The dust-extinction correction was performed assuming the uniformly mixed gas and dust. Here, 1 $\mathrm{R}$ (rayleigh) $=10^{6} / 4 \pi$ photons $\mathrm{cm}^{-2} \mathrm{~s}^{-1} \mathrm{sr}^{-1}$ and $1 \mathrm{CU}$ (continuum unit) $=$ photons $\mathrm{cm}^{-2} \mathrm{~s}^{-1} \mathrm{sr}^{-1} \AA^{-1}$. Galactic coordinates centered at $(l, b)=\left(0^{\circ}, 0^{\circ}\right)$ with longitude increasing toward the left are shown with latitude and longitude lines on a $30^{\circ}$ grid. 

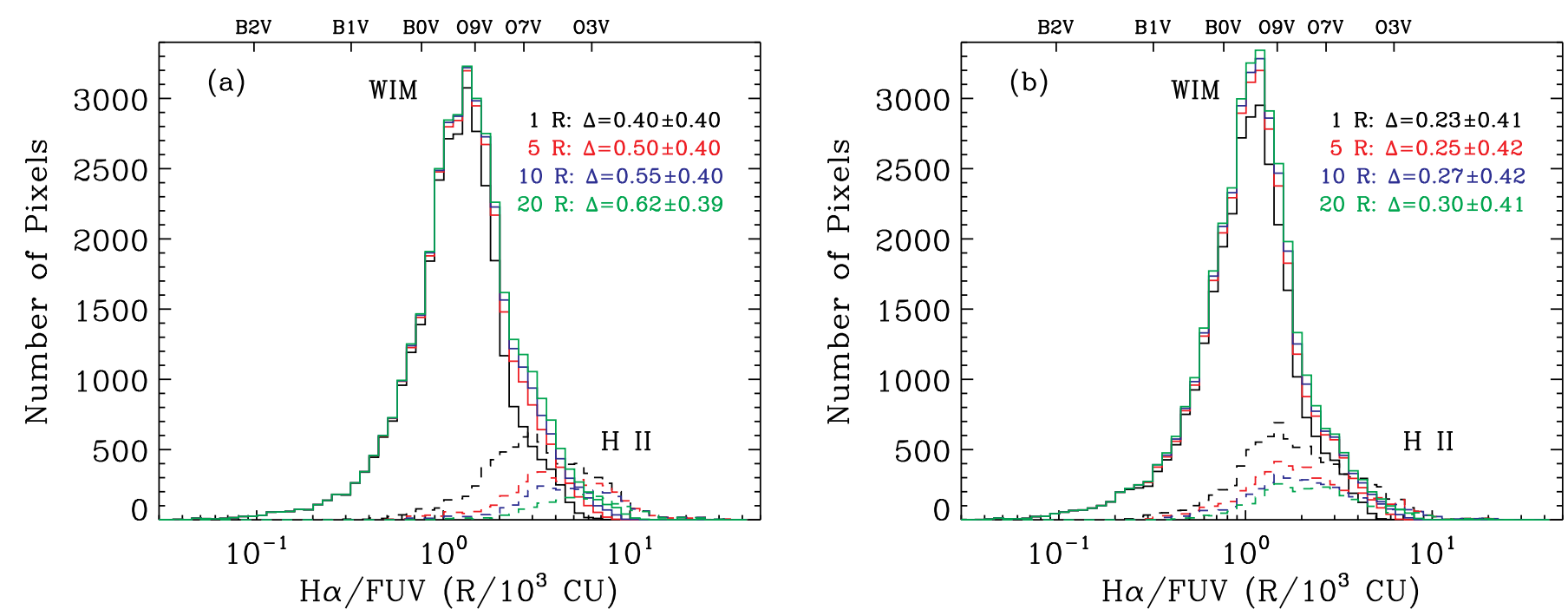

Figure 2. Histograms of the $\mathrm{H} \alpha / \mathrm{FUV}$ ratios for the $\mathrm{H}$ II regions and WIM. Extinction was (a) not corrected and (b) corrected assuming that the emitting gas is coextensive with dust. $\Delta$ indicates the difference of the mean intensity ratios in logarithmic scale for various thresholds. The ratios calculated for various stellar types are also indicated.
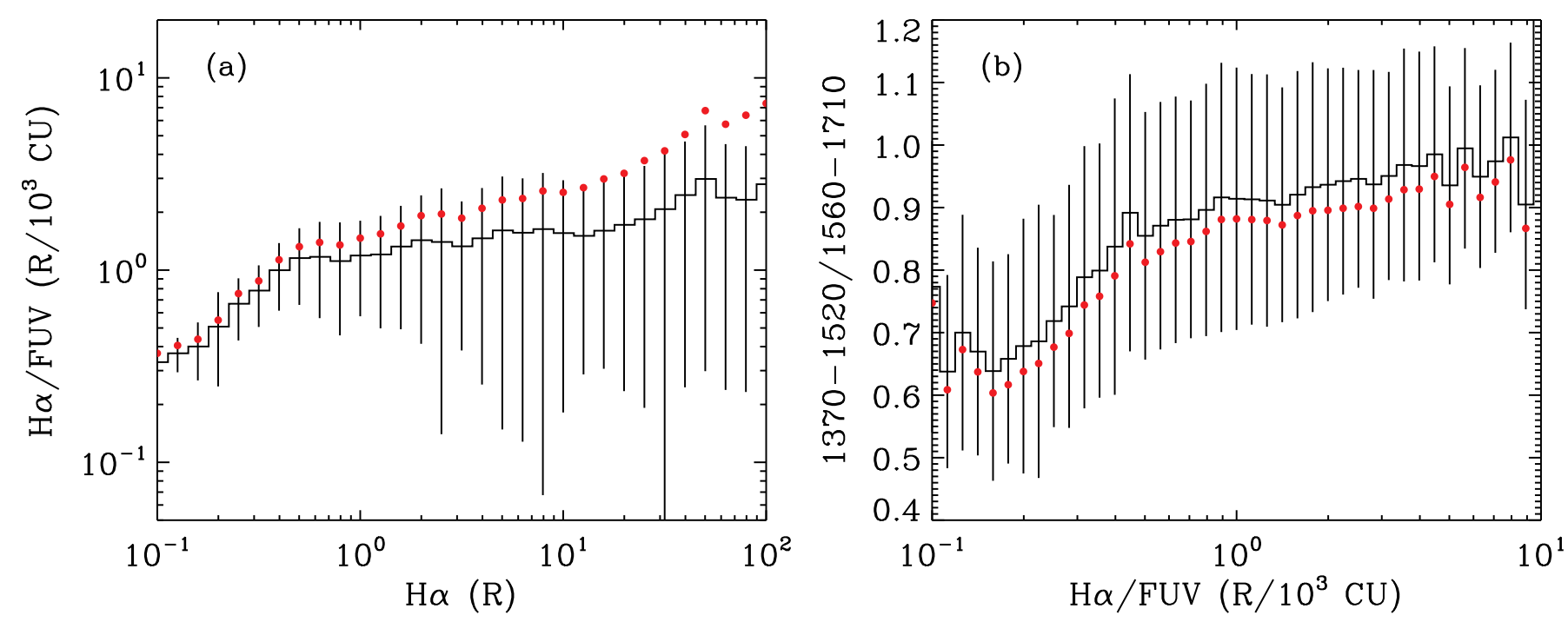

Figure 3. (a) The H $\alpha /$ FUV intensity ratio versus H $\alpha$ intensity. (b) The FUV Hardness ratio (1370-1520 to $1560-1710 \AA$ ) versus the H $\alpha /$ FUV intensity ratio. Black lines represent the average ( $\mathrm{H} \alpha / \mathrm{FUV}$ intensity or FUV hardness) ratios for given abscissa values after dust-extinction correction. Standard deviations of the $\mathrm{H} \alpha / \mathrm{FUV}$ ratios from its mean values are also shown. Red dots represent the ratios before the correction of dust-extinction. 

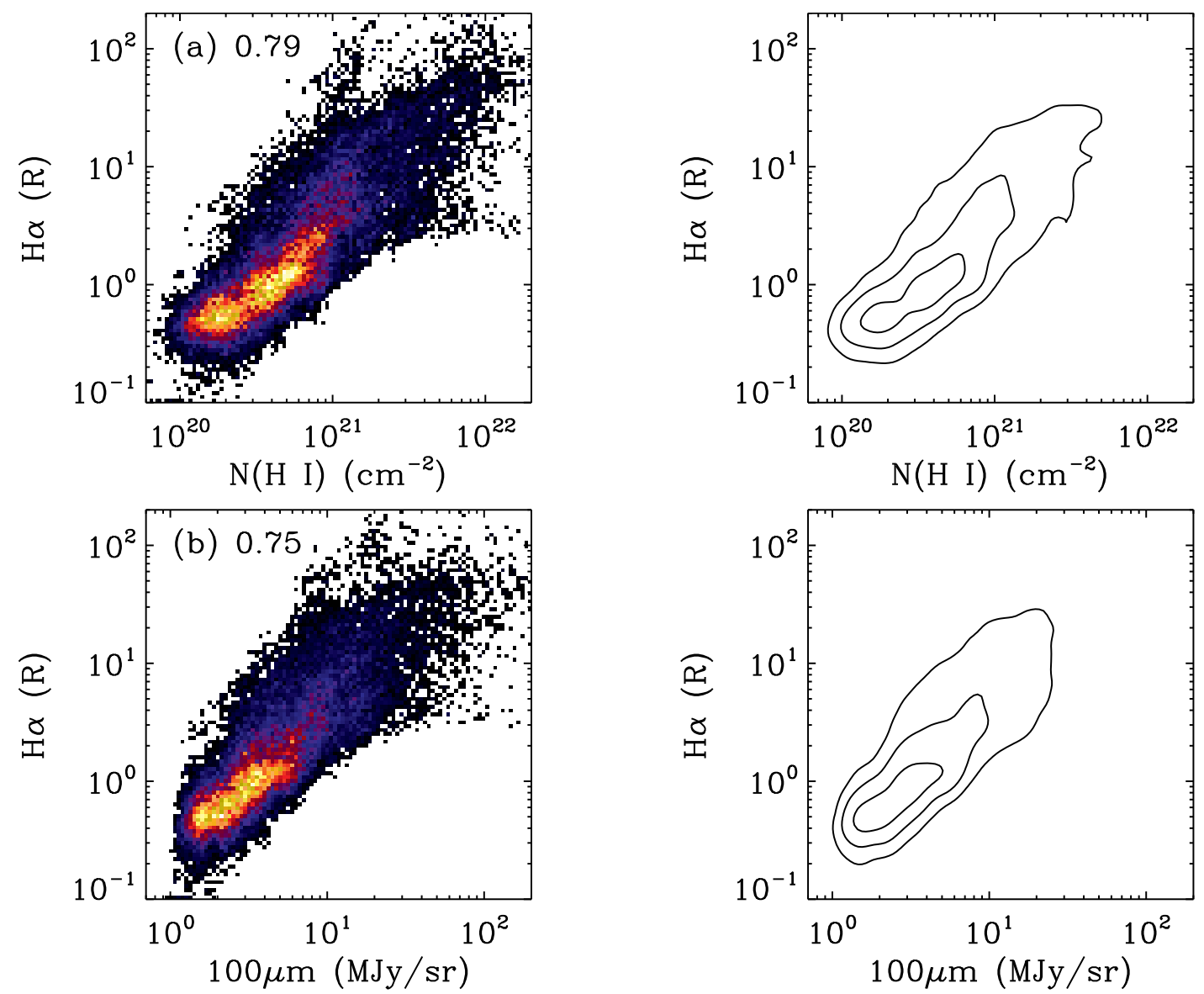

Figure 4. Correlation of the diffuse $\mathrm{H} \alpha$ background with (a) IR $100 \mu$ m emission, and (b) neutral hydrogen column density $N(\mathrm{H}$ I). The left panels show two-dimensional histogram and the right panels contours of the histograms. Numbers in the left panels are the correlation coefficients estimated in logarithmic scale. The contours correspond to 0.7, 0.3, and 0.1 of the maximum values of the histograms. See Figure 21(c) in Seon et al. (2011) for the comparison of the H $\alpha$ background with the FUV continuum background. The correlation coefficient of the H $\alpha$ versus FUV intensities is 0.81 from Figure 21(c) in Seon et al. (2011). 


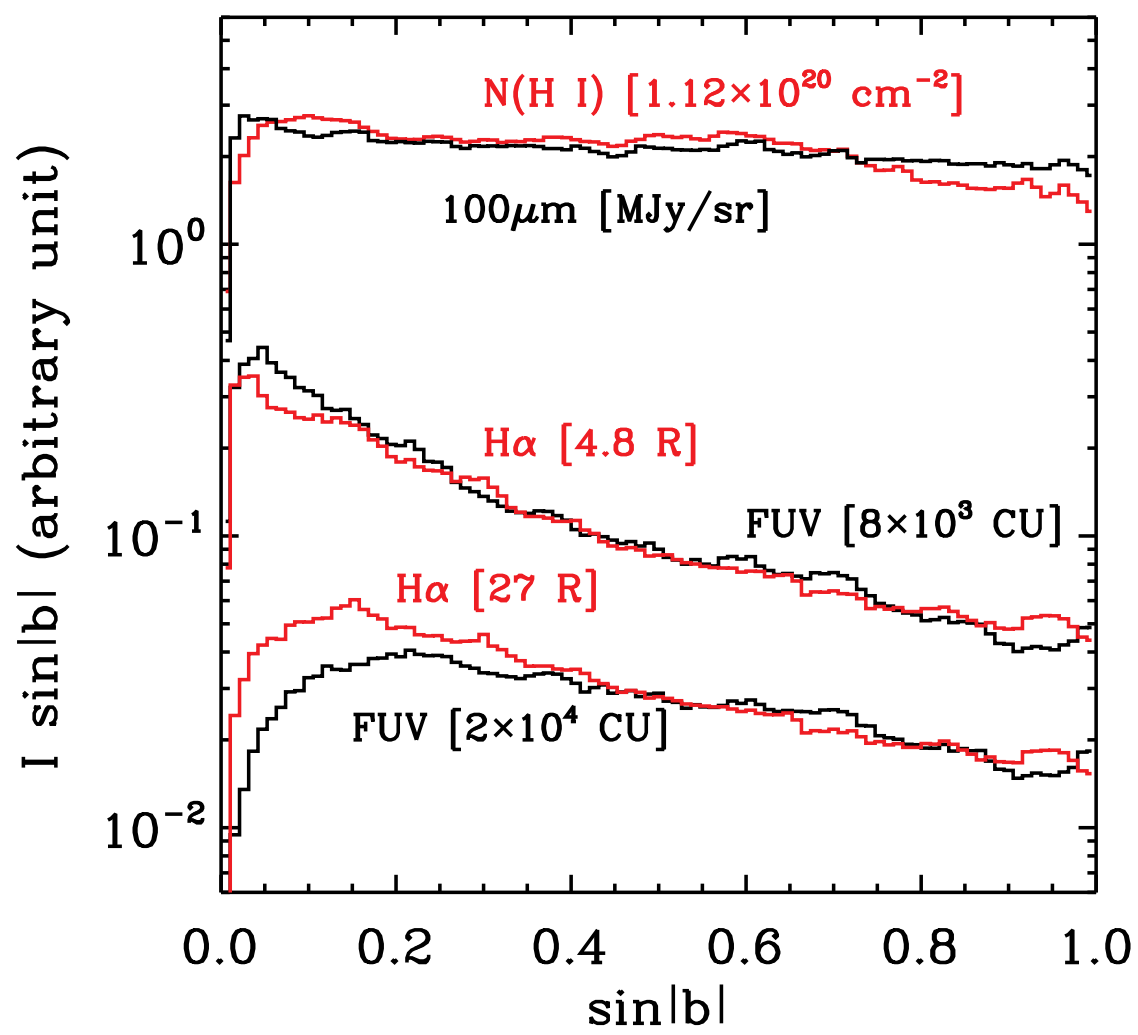

Figure 5. $I \sin |b|$ versus $\sin |b|$ for the neutral hydrogen column density $N(\mathrm{H} \mathrm{I}), 100 \mu \mathrm{m}$ emission, $\mathrm{H} \alpha$, and FUV intensities. Curves were shifted arbitrarily for comparison of the shapes. Top curves represent the variation of $N(\mathrm{H} \mathrm{I})$ and the $100 \mu \mathrm{m}$ emission in red and black colors, respectively. Middle and bottom curves show the variation of the $\mathrm{H} \alpha$ (red) and FUV (black) intensities. Middle curves represent the values after dust-extinction correction and bottom curves before the correction. Units are denoted in square brackets.

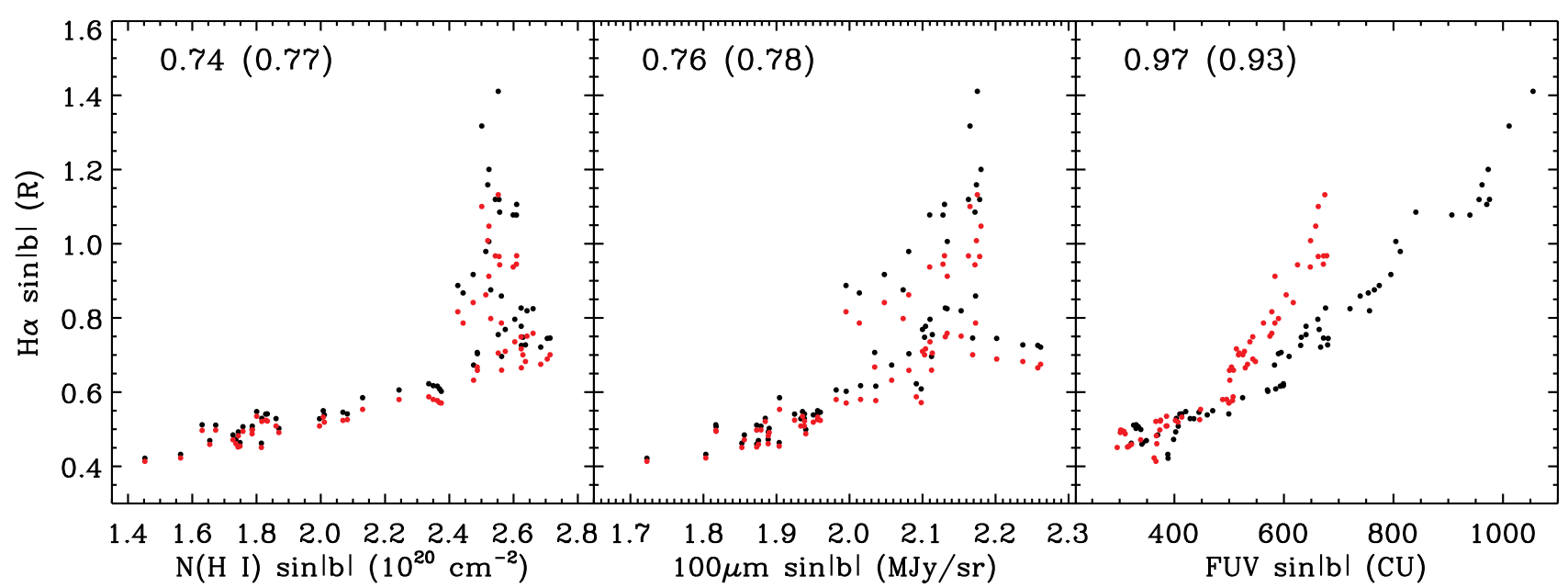

Figure 6. Correlation of the diffuse $\mathrm{H} \alpha$ intensity with neutral hydrogen column density (left), $100 \mu \mathrm{m}$ emission (middle), and the diffuse FUV intensity (right). Red and black dots represent the data points before and after the dust-extinction correction, respectively. Numbers outside and inside the parentheses are correlation coefficients estimated after and before the dust-extinction correction, respectively. 